\title{
Combined Detection of ACTN4 and SCC-Ag is a Promising Serological Biomarker for Cervical Intraepithelial Neoplasia 3 or Worse: A Case-Control Study
}

This article was published in the following Dove Press journal:

Risk Management and Healthcare Policy

\author{
Bin Zhu (D) \\ Binhua Dong $\mathbb{( D}^{2}$ \\ Simei Hong $\mathbb{1}^{3}$ \\ Meihua Wang (D) \\ Weichao Dai (D) ${ }^{4}$ \\ Qingzhu Zheng' \\ Dan $\mathrm{Wu}^{5}$ \\ Yingping Cao
}

'Department of Clinical Laboratorial Examination, Fujian Medical University Union Hospital, Fuzhou, People's Republic of China; ${ }^{2}$ Department of Gynecology, Laboratory of Gynecologic Oncology, Fujian Provincial Maternity and Children's Hospital, Affiliated Hospital of Fujian Medical University, Fuzhou, People's Republic of China; ${ }^{3}$ Department of Pathology, Fujian Provincial Cancer Hospital, Fuzhou, People's Republic of China; ${ }^{4}$ Department of Gynecology, Fujian Medical University Union Hospital, Fuzhou, People's Republic of China; ${ }^{5}$ Department of Pathology, Fujian Medical University Union Hospital, Fuzhou, People's Republic of China

Correspondence: Yingping Cao Department of Clinical Laboratorial Examination, Fujian Medical University Union Hospital, NO. 29, Xinquan Road, Fuzhou City, Fujian Province, People's

Republic of China

Tel +86-59l-86218678

Email caoyingping@aliyun.com

Binhua Dong

Department of Gynecology, Laboratory of Gynecologic Oncology, Fujian Maternity and Child Health Hospital, Affiliated Hospital of Fujian Medical University, NO. 18, Daoshan Road, Fuzhou, Fujian 35000I, People's Republic of China

Tel +86-59l-87558732

Fax +86-59।-8755I247

Email dbh |8-jy@|26.com
Purpose: Cervical cancer (CC) is a common malignancy in women. Squamous cell carcinoma antigen (SCC-Ag) and cancer antigen (CA)-125 are widely used to help diagnose CC, but novel tumour markers with superior sensitivity and specificity are needed. $\alpha$-Actinin 4 (ACTN4) is overexpressed in CC, though its diagnostic value for CC is unclear. This study examined the diagnostic value of ACTN4 and SCC-Ag as biomarkers for cervical intraepithelial neoplasia (CIN) 3 or worse.

Methods: Women screened for CC at Fujian Medical University Union Hospital were recruited from 2017.1 to 2018.5. Cervical tissues and blood were collected at the same time. Patients pathologically diagnosed as CIN3+ or NILM/CIN1/CIN2 were classified into the case and control groups, respectively. ACTN4 mRNA and protein levels were detected through quantitative PCR and immunohistochemistry, respectively, and ACTN4 and SCCAg concentrations were analysed by ELISA. The sensitivity, specificity, positive predictive value (PPV), negative predictive value (NPV), positive likelihood rate (PLR), negative likelihood rate (NLR), and Youden index (YI) of ACTN4 and SCC-Ag were evaluated. The optimum cut-off points for ACTN4 and SCC-Ag were determined by receiver operating characteristic (ROC) curve analysis, and accuracy was evaluated by the area under the ROC curve.

Results: In total, 105 patients were classified as CIN3+ cases and 106 as controls. The median ACTN4 levels in case and control tissues were 10.6 and 4.15, respectively. The ACTN4 and SCC-Ag concentrations were significantly higher in cases than controls $\left(\mathrm{P}_{\mathrm{ACTN}}\right.$ $\left.=0.0007 ; \mathrm{P}_{\mathrm{SCC}-\mathrm{Ag}}=0.0067\right)$. The sensitivity, specificity, PPV, NPV, PLR, NLR and YI of ACTN4 were $68.6 \%, 76.3 \%, 76.3 \%, 72.5 \%, 2.89,0.41$ and 44.9, respectively; SCC-Ag had a similar diagnostic value ( $\mathrm{P}>0.05)$, and ACTN4 combined with SCC-Ag had a superior diagnostic value $(75.6 \%, 87.5 \%, 88.6 \%, 73.7 \%, 6.05,0.28$, and 63.1 , respectively).

Conclusion: Combined ACTN4 and SCC-Ag detection is a promising serological biomarker for patients with CIN3 or worse.

Keywords: ACTN4, SCC-Ag, CIN3 or worse, serological biomarker

\section{Introduction}

Cervical cancer (CC) is the fourth most common cause of cancer in women worldwide and the most common cancer in women in eastern and middle Africa. Approximately $85 \%$ of the 528,000 new diagnoses of CC and $87 \%$ of the 266,000 deaths occur in less-developed regions all over the world. ${ }^{1}$ Persistent infection with 
one or more high-risk human papillomavirus (HR-HPV) types is closely associated with precancerous lesions and invasive cancer. ${ }^{2}$ The application of HPV DNA typing assays and cervical cytology has been undertaken in large numbers of hospital screenings for early cervical lesions. ${ }^{3}$ Among high-income countries, $\mathrm{CC}$ incidence and mortality have decreased by more than half over the past 30 years since the introduction of formal screening programmes. ${ }^{4}$ Unfortunately, malignancies of the cervix remain an important health issue in developing countries. ${ }^{1,4}$ Liquid-based cytology assays require shorter intervals and more experienced cytopathologists to ensure sensitivity and repeatability, ${ }^{5,6}$ which are lacking in developing countries. In contrast, asymptomatic women, especially elderly women, sometimes lack testing budgets and have little willingness to have exfoliated cells collected from the cervix, which is invasive and cannot be repeatedly collected in a short period of time. Thus, extensive screening for early cervical lesions using the HR-HPV test and cytological detection is difficult. Additionally, notwithstanding that late-stage patients have undergone extensive surgeries and systemic medical treatments, their recovery remains unpromising. Currently, squamous cell carcinoma antigen (SCC$\mathrm{Ag}$ ) and cancer antigen (CA)-125 are helpful to diagnose patients with $\mathrm{CC}$ and have been widely used in clinical laboratories, but new serological tumour markers with superior sensitivity and specificity suitable for screening early cervical lesions still need to be identified.

$\alpha$-Actinin 4 (gene name ACTN4) is an actin crosslinking protein composed of 911 amino acids that belongs to the spectrin superfamily and was first identified in 1998. ACTN4 is ubiquitously expressed in normal non-muscle cells, and this protein is involved in regulating the binding of actin fibres by interacting with cell membranes. Additionally, this protein plays an essential role in regulating cellular signalling pathways correlated with various types of cancer progression and poor patient prognosis, which are involved in the invasion and metastasis of various cancer cells, such as colorectal cancer, pancreatic cancer, and ovarian cancer. ${ }^{9-11}$ In particular, ACTN4 plays an important role in the epithelial-to-mesenchymal transition, cancer stem cell properties and chemoresistance in $\mathrm{CC},{ }^{12-14}$ indicating that it may facilitate cell migration and invasion. In addition, HPV16 E6, a famous HR-HPVrelated oncoprotein, is involved in $\mathrm{CC}$ cell migration and invasion via ACTN4. ${ }^{15}$ Furthermore, it has been reported that ACTN4 from cervicovaginal fluid can discriminate patients with $\mathrm{CC}$ and patients infected by HR-HPV, ${ }^{16-18}$ and ACTN4 mRNA in serum is elevated in patients with breast cancer. ${ }^{19}$ Therefore, ACTN4 in blood may have potential screening abilities for early cervical lesions.

SCC-Ag was first described by Kato and Tarigoe (1977) in neutral and acidic sub-fractions of tumour antigen 4 (TA-4). Squamous cell cervical carcinomas constitute $85-90 \%$ of all cervical carcinomas. Elevated serum SCC levels have been detected in $28-88 \%$ of cervical squamous cell carcinomas. ${ }^{20}$ The study of two or more biological markers in serum to increase the sensitivity and specificity of early screening of malignant tumours by making up their own blind spots and increasing their own superior detection ability has remained a research hotspot for generations of scholars.

In this study, we measured the levels of ACTN4 and SCC-Ag in 211 patients from NILM to CC to analyse their single or combined value in the diagnosis of early cervical lesions using a CIN3 cut-off. Meanwhile, we identified the relative levels of ACTN4 mRNA and protein in tissue to evaluate the expression of these biomarkers in $\mathrm{CC}$ tissues with different disease severity. Furthermore, we assessed the relationship between the relative expression of ACTN4 mRNA and clinicopathologic characteristics in patients with CC. Our study suggested that ACTN4 combined with SCC-Ag in serum has a potential role as a biomarker for the diagnosis and prognosis of $\mathrm{CC}$.

\section{Materials and Methodology Patients and Study Design}

This retrospective cohort consisted of healthy patients undergoing routine gynaecological physical examinations at a medical examination centre and inpatients who underwent colposcopy biopsy and radical hysterectomy at Fujian Medical University Union Hospital from January 2017 to May 2018. Among the participants, the healthy individuals selected during annual health examinations showed no evidence of disease; the CIN patients were diagnosed by colposcopy and histology; and the invasive CC patients had stage I to II disease based on the International Federation of Gynecology and Obstetrics (FIGO) classification and were recruited after the histopathologic confirmation of CC tumours. After obtaining informed written consent, punch biopsy or surgically resected cervical tissues were collected from all the enrolled patients during their diagnostic/treatment procedures. The demographic and clinicopathological characteristics were recorded from clinical data and in-person 
interviews. In addition, blood samples (by venous puncture) were also obtained from the same cohort of patients. A study flowchart is shown in Figure 1

\section{Processing of Tissue and Blood Samples}

Fresh cervical tissues were divided into two parts after biopsy/surgical resection. One part of the tissue was immediately stored in liquid nitrogen after washing with physiological saline to prepare for qPCR assays. The other piece of tissue was fixed in $10 \%$ buffered formalin and then embedded in paraffin to prepare tissue blocks that were cut into 5 - $\mu \mathrm{m}$ sections and placed on poly-L-lysinecoated glass slides. Tissue architecture and the usefulness of sections for immunohistochemical analysis were

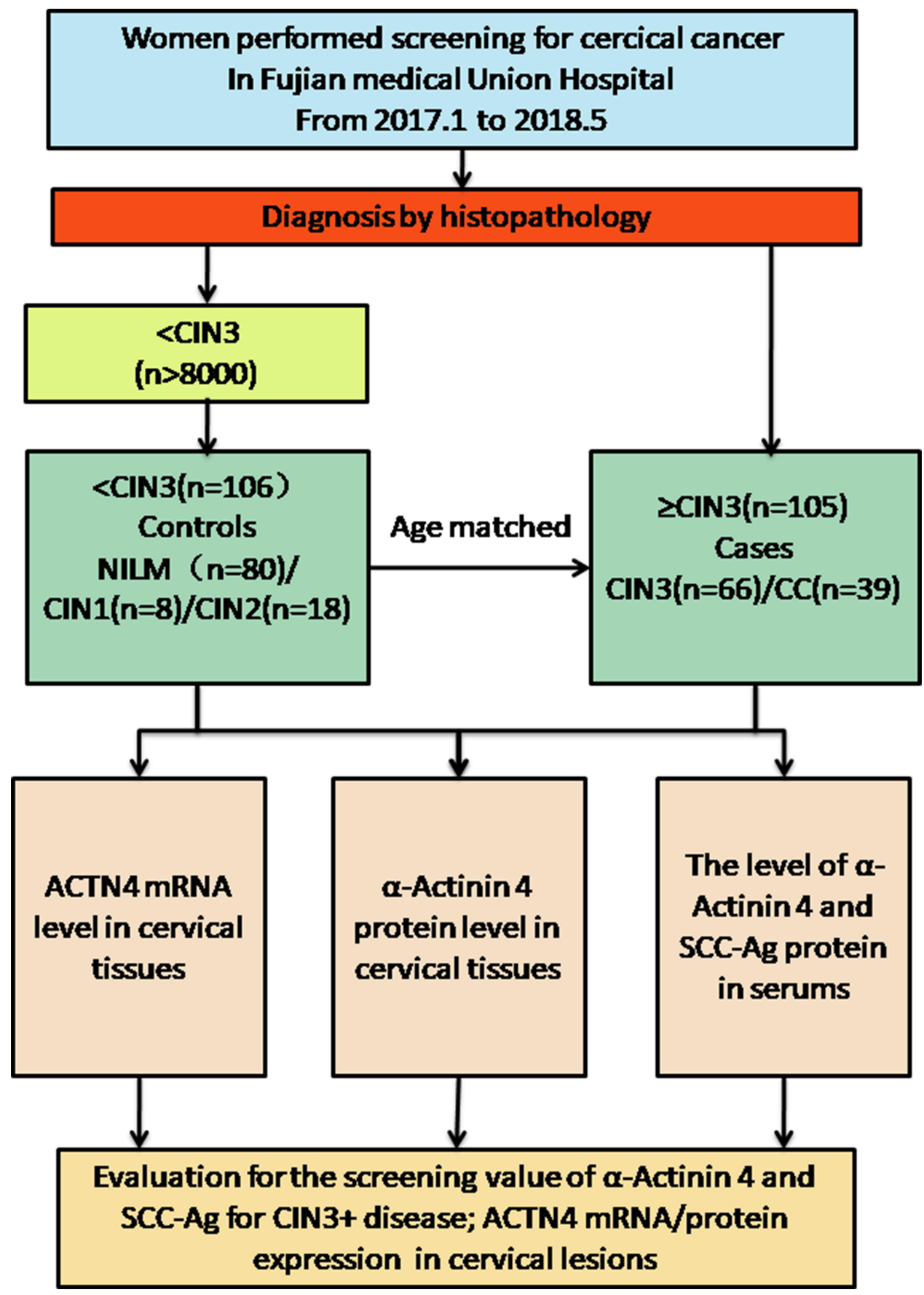

Figure I Flowchart of the screening profiles of women in the case-control study.

Abbreviations: NILM, negative for intraepithelial lesion or malignancy; CIN, cervical intraepithelial neoplasia; CC, cervical cancer; SCC-Ag, squamous cell carcinoma antigen. 
initially evaluated by routine histological staining. Slides confirmed with haematoxylin and eosin (H\&E) were further processed for IHC with ACTN4 staining. The collected blood samples were centrifuged at $1500 \mathrm{~g}$ for $10 \mathrm{~min}$ to separate the serum. Serum samples were then aliquoted and stored at $-70^{\circ} \mathrm{C}$ for ELISA.

\section{ACTN4 mRNA Expression in Cervical Cancers and Control Tissues}

Total RNA was isolated from frozen tissues with the TRIZOL reagent (Invitrogen, NY, USA) according to the manufacturer's instructions. The RNA concentration and purity were measured by a UV spectrophotometer (GeneBio, Inc, America). RNA was reverse-transcribed with a high-capacity cDNA Reverse transcription kit (TAKARA, Japan). The aliquots of double-stranded cDNA were amplified using qRT-PCR with a SYBR Green PCR Kit (TAKARA, Japan). qRT-PCR was performed using the following primers for ACTN4 (forward 5'-TTGATGAGG ACTTCCGAGACG-3' and reverse 5'-TGGCGAACCTAA GGATGATGG-3') and GAPDH (forward 5'-CACATGGC CTCCAAGGAGTA3 and reverse 5'-TGAGGGTCTCT CTCTTCCTCTTGT-3'). Calculations to determine the relative gene expression level were performed using the cycle threshold $(\mathrm{Ct})$ method. The mean $\mathrm{Ct}$ values from triplicate measurements were used to calculate the relative expression of ACTN4 and were normalized to GAPDH using the $2^{-\Delta \Delta C t}$ method. We calculated the $\Delta \Delta \mathrm{Ct}$ using the maximum $\Delta \mathrm{Ct}$ value among all samples as a positive control.

\section{ACTN4 Expression in Cervical Tissues with Different Disease Severity}

For immunohistochemistry (IHC), sections were deparaffinized in xylene, rehydrated in a serial ethanol gradient, blocked with $3 \% \mathrm{H}_{2} \mathrm{O}_{2}$ for $10 \mathrm{~min}$, and then incubated at $100^{\circ} \mathrm{C}$ in water with $10 \mathrm{mM}$ citric sodium $(\mathrm{pH}$ 6.0) for $30 \mathrm{~min}$ to retrieve the antigens. Then, the sections were incubated with a rabbit monoclonal anti-ACTN4 (7H6 ab32816;1:100; Abcam, Cambridge, United Kingdom) primary antibody at $4^{\circ} \mathrm{C}$ overnight. A secondary antibody conjugated with horseradish peroxidase was applied for $30 \mathrm{~min}$ at room temperature, followed by development with a diaminobenzidine solution. For the negative control, primary antibodies were replaced with phosphatebuffered saline. We used a colon cancer tissue section as the positive control for ACTN4. The IHC results from ACTN4 staining were independently examined by two deputy chief pathologists. Only cytoplasmic immunopositivity was considered positive for ACTN4 expression. The scoring criterion for semi-quantification was determined by multiplying the staining intensity score [negative $(0)$, mild $(+1)$, moderate $(+2)$, and intense $(+3)]$ and the percentage $(\%)$ of positively stained cells [negative $(0),<5 \%$ positively stained cells $(+1), 5 \%-20 \%$ cell positivity $(+2), 21 \%-50 \%$ cell positivity $(+3)$, and $>50 \%$ cell positivity $(+4)]$. Based on the calculated IHC score, the samples were further divided into mildly positive (IHC scores of 1 and 2), moderately positive (IHC scores of 3 , 4, and 6), and intensely positive (IHC scores of 8, 9, and 12) groups.

\section{The Expression of ACTN4 in Serum with Different Cervical Severity}

The serum concentration of ACTN4 was measured by a quantitative sandwich enzyme immunoassay with a commercially available ELISA kit (No: CSB-E13742h; CUSABIO, Biotech Co., Ltd, Wuhan, China) according to the manufacturer's instructions. The detection range for serum ACTN4 was 31.25-2000 pg/mL with a sensitivity of $7.8 \mathrm{pg} / \mathrm{mL}$. The optical density (OD) value of each well was measured by a microplate reader at $450 \mathrm{~nm} 5 \mathrm{~min}$ after termination of the reaction. Using OFFICE EXCEL (2010 version), the highest two points of the standard were discarded, a standard curve was drawn using the binomial equation, and the corresponding sample concentrations were calculated. A standard curve was generated from different sample dilutions and standard product mixtures according to the manufacturer's website. SCC-Ag (squamous cell carcinoma antigen) was measured using a chemiluminescent microparticle immunoassay (CMIA) kit according to the manufacturer's instructions (SCC8D18; Architect, DENKA SEIKEN CO, LTD. Tokyo, Japan).

\section{Statistical Analysis}

Because the Shapiro-Wilk test for normality indicated that the relative expression of ACTN4 mRNA $\left(2^{-\Delta \Delta \mathrm{ct}}\right)$ and the serum concentrations of ACTN4 were not normally distributed, the Mann-Whitney test was applied to investigate the significance of differences in ACTN4 mRNA levels for each clinicopathologic variable or different histologic diagnosis. Welch's test was applied to analyse the ACTN4 protein levels in tissue and serum of patients with different cervical severity. Pearson's correlation coefficient (r) was used to determine the association of ACTN4 
expression between tissue histoscores and serum concentrations. Receiver operating characteristic (ROC) curve analysis was performed to determine the sensitivity, specificity, positive predictive value (PPV), negative predictive value (NPV), positive likelihood rate (PLR), negative likelihood rate (NLR), and Youden index (YI) of ACTN4 and SCC-Ag tests according to the standard definitions for CIN3+. The AUC (area under the ROC curve) of ACTN4 in combination with the SCC-Ag ELISA was analysed by binary logistic regression to calculate a combined predictive factor by a logistic regression equation. The sensitivity, specificity, PPV, NPV, PLR, NLR, and YI of the combined test were considered positive when ACTN4 or SCC-Ag exceeded the respective cut-off value. All data analyses were performed using SPSS 17.0 (SPSS Inc., Chicago, IL, USA) and GraphPad Prism 8.0.1 (www.graph pad.com). All statistical tests were two-sided, and a P-value $<0.05$ was considered to indicate statistical significance.

\section{Results}

\section{Patients}

After obtaining the above-mentioned tissue and serum samples, 39 frozen tissues from CC and 39 control tissues were analysed by qPCR to determine the relative expression of ACTN4 mRNA, and 211 tissues embedded in paraffin and serum were used for immunohistochemistry (IHC)/enzymelinked immunosorbent assay (ELISA) to examine ACTN4 protein expression. Negative for intraepithelial lesion or malignancy (NILM), LSIL (CIN1), HSIL (high-grade squamous intraepithelial lesion, CIN2/3) and CC, including NILM $(\mathrm{n}=80)$ and CIN1 $(\mathrm{n}=8) / \mathrm{CIN} 2(\mathrm{n}=18)$ were used as controls, while $\mathrm{CIN} 3(\mathrm{n}=66) / \mathrm{CC}(\mathrm{n}=39)$ were used as cases. Clinically, $17.9 \%$ patients were diagnosed with squamous cell carcinoma (SCC) FIGO IA, 35.8\% were diagnosed with SCC FIGO IB, $39.7 \%$ were diagnosed with SCC FIGO IIA, and $6.6 \%$ patients were diagnosed with cervical adenocarcinoma (ADC). The median age of CIN patients was 47 years (range 21-72 years), the median age of $\mathrm{CC}$ patients was 55 years (range 23-85 years), and the median age of CIN patients was 50 years (range 21-80 years). Among CC patients, HPV type 16 (type18 negative) infections were noted in $60.6 \%$ of patients, while HPV type18 (type16 negative) infections were found in $9.1 \%$ of patients, and HPV type non-16/18 were found in $30.3 \%$ of patients. Lymph node positivity was noted in $33.3 \%$ of CC patients, while $66.6 \%$ of patients did not exhibit lymphatic involvement.

\section{ACTN4 mRNA Expression in Cervical Tissues with Different Disease Severity and Correlation Between ACTN4 mRNA Expression and CC Patient Clinical \\ Parameters}

To examine ACTN4 expression, we isolated total RNA from $\mathrm{CC}$ tissues and control tissues and found that the relative levels of ACTN4 in CC based on qPCR were significantly higher than in the control (10.6 vs $4.15, \mathrm{P}=0.0074)$. To further investigate whether ACTN4 expression levels were correlated with clinicopathological variables, a correlation of ACTN4 expression with clinicopathological data from CC patients was performed. The results revealed that the relative levels of ACTN4 increased as the FIGO stages, tumour size, and lymph node metastasis increased (12.12 vs 4.1, $\mathrm{P}=0.003$; 16 vs $8.4, \mathrm{P}=0.0183$ and 12.13 vs $5.1, \mathrm{P}=0.033$, respectively). No relationship was found between ACTN4 and other clinicopathological parameters, such as age (patients older or younger than 55 years old), HPV type (16/non-16; 16, 18/ non-16, 18) and growth direction of invasive cancer $(\mathrm{P}=0.96$, $\mathrm{P}=0.07, \mathrm{P}=0.07$, and $\mathrm{P}=0.43$, respectively) (Figure 2).

\section{ACTN4 Protein Expression in the Tissue and Serum of Patients with Different Cervical Lesion Severities}

With the addition of mRNA specimens collected from $\mathrm{CC}$ and control tissues, we further investigated ACTN4 protein levels in tissue and serum from a continuous cohort that included 106 control women and 105 patients with CIN3+. There was a gradual increase in ACTN4 histoscores from cervical tissue in controls and CIN3+ $(0.86 \pm 1.03$ vs $2.85 \pm 1.70, \quad \mathrm{P}<0.0001)$. Similarly, the increase in ACTN4 concentrations $(\mathrm{pg} / \mathrm{mL})$ in serum from controls to cases $(28.51 \pm 11.30$ vs $43.70 \pm 23.60$, $\mathrm{P}=0.0007)$ is well known. Moreover, serological SCC$\mathrm{Ag}$ concentrations $(\mathrm{ng} / \mathrm{mL})$ in controls and cases were significantly different $(0.93 \pm 0.44$ vs $4.15 \pm 7.07$, $\mathrm{P}=0.0067$ ) (Table 1). In addition, out of $84 \mathrm{CIN} 2 / 3$ patients, $43(50.8 \%)$ patients showed cytoplasmic overexpression of ACTN4 protein (Figure 3). Among these, the expression was moderate in $68.8 \%$ of patients (IHC scores of 3, 4, and 6) and mild in $31.1 \%$ of patients (IHC scores of 1 and 2). Similarly, among CC patients, 29/39 (74.4\%) patients exhibited significant overexpression of ACTN4 (Figure 3). Out of these 39 patients, mild ACTN4 staining was detected in 2 patients (IHC scores 

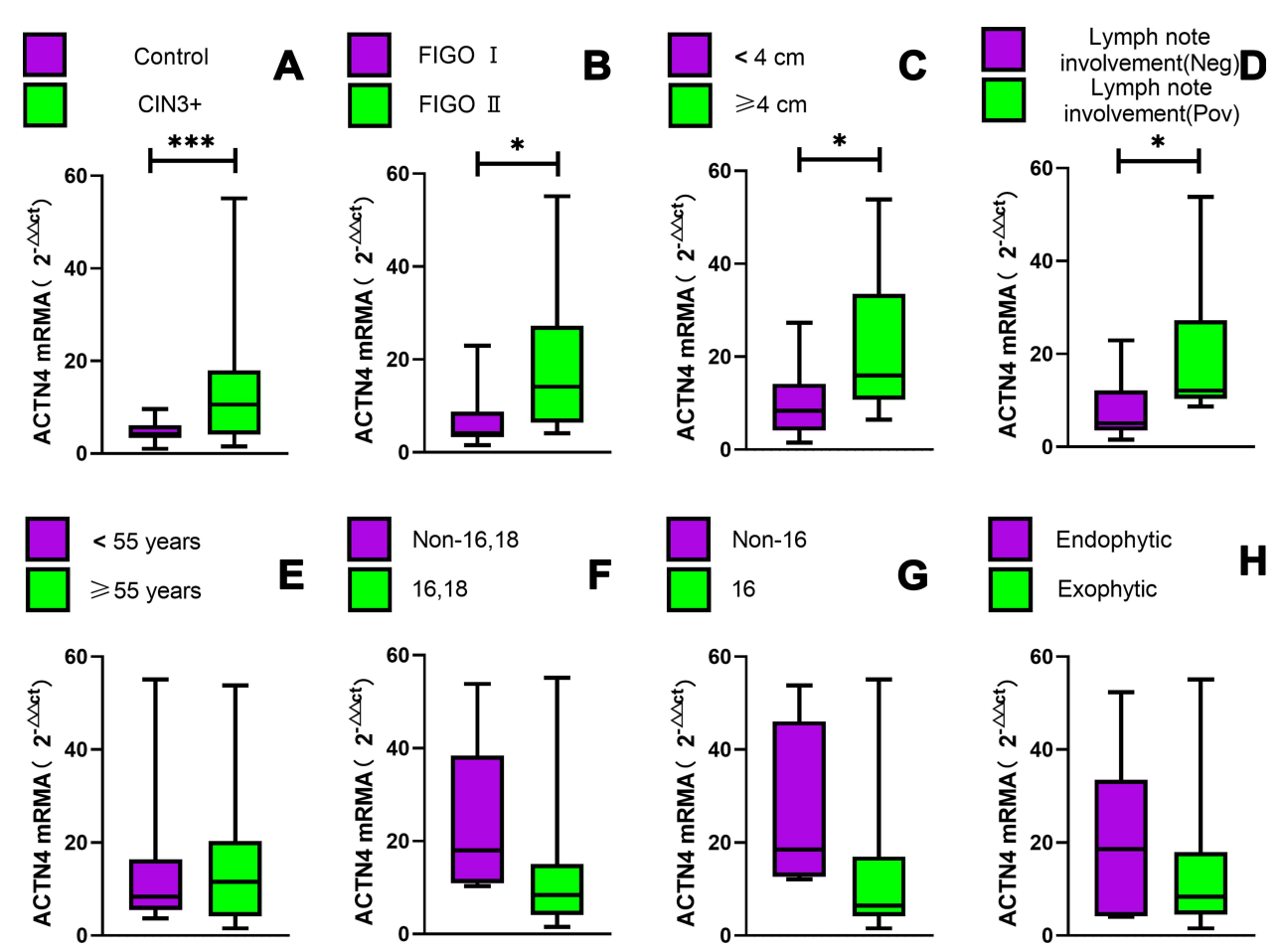

Figure 2 The relative expression of ACTN4 mRNA in cervical tissues with different cervical severity and clinicopathologic variables. (A-H) The Mann-Whitney test was used to analyse significant differences between the purple and green groups; (B-H) ACTN4 mRNA was detected in women with CC. $* \mathrm{P}<0.05$, $* * * P<0.00 \mathrm{I}$.

Abbreviation: FIGO, International Federation of Gynecology and Obstetrics.

of 1 and 2), moderate staining was detected in 30 patients (IHC scores of 3, 4, and 6), and intense staining was observed in 7 patients (IHC scores of 8, 9, and 12). Endogenous ACTN4 expression was not observed in the majority of normal/CIN1 cervix tissues and was only observed in 7 patients with IHC scores of 1 and 2 and 6 patients with IHC scores of 3 who showed very mild but detectable reactivity. In contrast, strong cytoplasmic protein immunopositivity was observed in both HSIL (CIN2/3) and CC patients compared with normal/CIN1 patients (Figure 3).
Performance of Different Serological Biomarkers for Identifying CIN3+ Disease and the Correlation of ACTN4 Expression Between Cervical Tissues and Serum

We analysed serological biomarker levels using ROC curve models in a cohort that ranged from NILM to CC and evaluated the performance of ACTN4, SCC-Ag, and ACTN4 in combination with SCC-Ag in serum to identify CIN3+ disease. According to the ROC curve analysis, $\mathrm{AUC}_{\text {combination }}$

Table I $\alpha$-Actinin 4 and SCC-Ag Expression in Tissues and Sera from Control and CIN3+ Patients

\begin{tabular}{|c|c|c|c|c|c|c|}
\hline Location & Cervical Pathology & No. of Women & $\begin{array}{l}\text { IHC Scores and Serological } \\
\text { Concentration }(\mathrm{pg} / \mathrm{mL}) \text { of } \\
\alpha \text {-Actinin } 4\end{array}$ & $P$ value & SCC-Ag (ng/mL) & $P$ value \\
\hline Tissue & $\begin{array}{l}\text { Control } \\
\text { CIN3+ }\end{array}$ & $\begin{array}{l}106 \\
105\end{array}$ & $\begin{array}{l}0.86 \pm 1.03 \\
2.85 \pm 1.70\end{array}$ & $<0.0001^{\mathrm{a}}$ & $\begin{array}{l}\rho^{\mathrm{b}} \\
\rho^{\mathrm{b}}\end{array}$ & \\
\hline Serum & $\begin{array}{l}\text { Control } \\
\text { CIN3+ }\end{array}$ & $\begin{array}{l}106 \\
105\end{array}$ & $\begin{array}{l}28.51 \pm 11.30 \\
43.70 \pm 23.60\end{array}$ & $0.0007^{\mathrm{a}}$ & $\begin{array}{l}0.93 \pm 0.44 \\
4.15 \pm 7.07\end{array}$ & $0.0067^{\mathrm{a}}$ \\
\hline
\end{tabular}

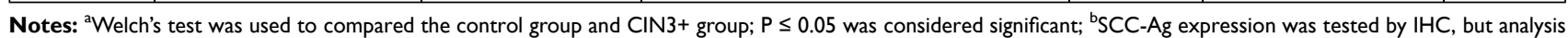
by pathologists cannot be performed due to a lack of cases after the detection of $\alpha$-Actinin 4 . Because SCC-Ag is overexpressed in cervical cancer tissues compared with normal cervical tissues and this has been reported in many studies, the detection of SCC-Ag was mainly used to compare the concentration of $\alpha$-Actinin 4 in serum. Abbreviations: NILM, negative for intraepithelial lesion or malignancy; CIN, cervical intraepithelial neoplasia; CC, cervical cancer; SCC-Ag, squamous cell carcinoma antigen; Control: patients with NILM/CINI/CIN2 are considered as control. 

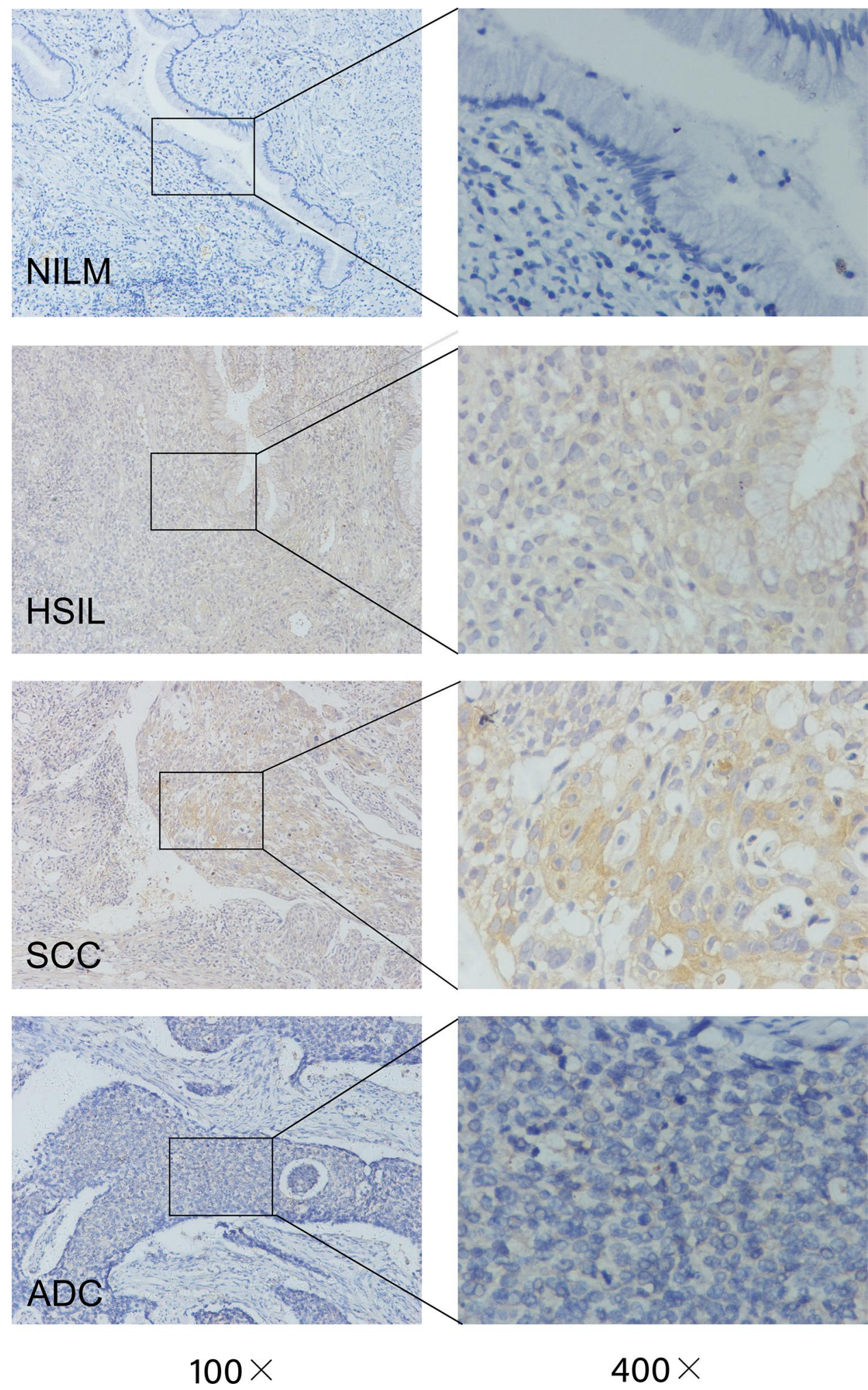

Figure 3 IHC staining for ACTN4 in cervical lesion tissues with different disease severities.

Abbreviations: HSIL, high-grade squamous intraepithelial lesion; SCC, squamous cervical cancer; ADC, cervical adenocarcinoma.

was superior to $\mathrm{AUC}_{\mathrm{ACTN} 4}$ and $\mathrm{AUC}_{\mathrm{SCC}-\mathrm{Ag}}(81.4,72.0$ and 75.4, respectively). In addition to logistic regression analysis, we calculated the screening values of different biomarkers based on the respective cut-off point, which demonstrated that there were increases in ACTN4, SCC-Ag and ACTN4 in combination with SCC-Ag in terms of sensitivity (68.6, 
68.6 and 75.6), specificity (76.3, 86.8 and 87.5), PPV (72.7, 82.8 and 88.6), PLR (2.89, 5.20 and 6.05), and YI (44.9, 55.4 and 63.1). Meanwhile, there was a decrease in the NLR $(0.41$, 0.37 , and 0.28$)$ and a steady decrease in the NPV $(72.5,75.0$, and 73.7) (Table 2). A cut-off value of $33.07 \mathrm{pg} / \mathrm{mL}$ for the serological ACTN4 assay can be used to screen for CIN3+ diseases. According to the correlation analysis, we found that ACTN4 levels in tissue and serum showed a significant positive correlation $(\mathrm{r}=0.19, \mathrm{P}=0.006)$ (Figure 4).

\section{Discussion}

Great effort has been made by scientists to identify one or more biomarkers that can predict invasive cancer as early as possible. Serologic biomarkers may appear in the surrounding tissue and be released into the blood during cervical lesion growth, which has some advantages. These biomarkers are economical, quantitative, and minimally invasive. ${ }^{20}$ In addition, we can obtain multiple specimens at the same time, and the tests do not require expensive instruments, licensed PCR laboratories, or experienced pathologists, which are difficult to find in less developed regions. DNA typing assays and cervical cytology are common detection methods for screening of early cervical lesions, but they require shorter intervals and more experienced cytopathologists. ${ }^{5,6}$ ACTN4 plays an important role in the proliferation and invasion of various cancers. Therefore, we hypothesized that actinin, which participates in the binding of actin fibres and tumour invasion, may be useful in the early detection of cancerous changes. In this case-control study, we found that ACTN4 levels in serum were significantly elevated in patients with CIN3 or worse. When we detected SCC-Ag levels, we found thatACTN4 in serum had a similar diagnostic value as SCC-Ag. When ACTN4 was detected in combination with SCC-Ag, we found a superior sensitivity and specificity over single detection ofACTN4 or SCCAg. Moreover, the levels of ACTN4 mRNA and protein were elevated in tissues with cervical lesion severity, and the levels of ACTN4 mRNA were significantly associated with FIGO stage, tumour size, and lymph node metastasis.

ACTN4 is ubiquitously expressed in normal nonmuscle cells, and this protein is involved in regulating the binding of actin fibres by interacting with cell membranes. ${ }^{8}$ Results from recent studies revealed that ACTN4 plays an important role in the progression of various cancers and in poor patient prognosis. Moreover, ACTN4 may not only participate in the epithelial-tomesenchymal transition and tumorigenesis of $\mathrm{CC}$, but is also associated with carcinogenesis through the E6 protein, a famous HR-HPV oncoprotein. ${ }^{12-15}$ Nevertheless, studies about the association between ACTN4 and CC are limited, and the value of serological ACTN4 screening for early cervical lesions is not fully understood. Thus, it is necessary to further investigate the value of ACTN4 in the diagnosis of $\mathrm{CC}$ in populations with different cervical lesion severity. To our knowledge, ACTN4 has a potential screening capacity for early cancer, including $\mathrm{CC}$, in fluids and bloods collected from the human body. Comparable results for ACTN4 were obtained by Starodubtseva, N. L, ${ }^{16}$ Geert A. A. Van Raemdonck ${ }^{17}$ and Van Ostade $\mathrm{X},{ }^{18}$ where elevatedACTN4 levels were found in the cervicovaginal fluid of $\mathrm{CC}$ patients against NILM and patients infected by HR-HPV against LR-HPV. Moreover, Cheng Fang ${ }^{19}$ reported that ACTN4 mRNA levels detected by qPCR can serve as a clinical predictor for the diagnosis of clinical outcomes of patients with breast cancer (BC), and the potential ability to screen for early cervical lesions is consistent with our findings.

Squamous cell cervical carcinomas (SCC-Ag) constitute $85-90 \%$ of all cervical carcinomas. ${ }^{20}$ Elevated serum

Table 2 Diagnosis Values of Serum $\alpha$-Actinin 4, SCC-Ag and Combination for Cervical Cancer

\begin{tabular}{|l|l|l|l|}
\hline Biomarker (CIN3 ${ }^{+}$Endpoint) & $\alpha$-Actinin 4 & SCC-Ag & $\begin{array}{l}\boldsymbol{\alpha}-A c t i n i n ~ 4 \text { in Combination } \\
\text { With SCC-Ag }\end{array}$ \\
\hline Sensitivity\% (95\% Cl) & $68.6(50.6-82.6)$ & $68.6(50.6-82.6)$ & $75.6(59.3-87.1)$ \\
Specificity\% (95\% Cl) & $76.3(59.4-87.9)$ & $86.8(68.2-94.5)$ & $87.5(70.1-95.9)$ \\
PPV\% (95\% Cl) & $72.7(54.2-86.1)$ & $82.8(63.5-93.5)$ & $88.6(72.3-96.3)$ \\
NPV\% (95\% Cl) & $72.5(55.9-84.9)$ & $75.0(55.9-84.9)$ & $73.7(56.6-86.0)$ \\
PLR\% (95\% Cl) & $2.89(1.57-5.35)$ & $5.20(2.01-10.80)$ & $6.05(2.38-15.38)$ \\
NLR\% (95\% Cl) & $0.41(0.25-0.68)$ & $0.37(0.22-0.61)$ & $0.28(0.16-0.48)$ \\
\hline Yl\% & 44.9 & 55.4 & 63.1 \\
\hline
\end{tabular}

Abbreviations: PPV, positive predictive value; NPV, negative predictive value; PLR, positive likelihood rate; NLR, negative likelihood rate; YI, Youden index. 

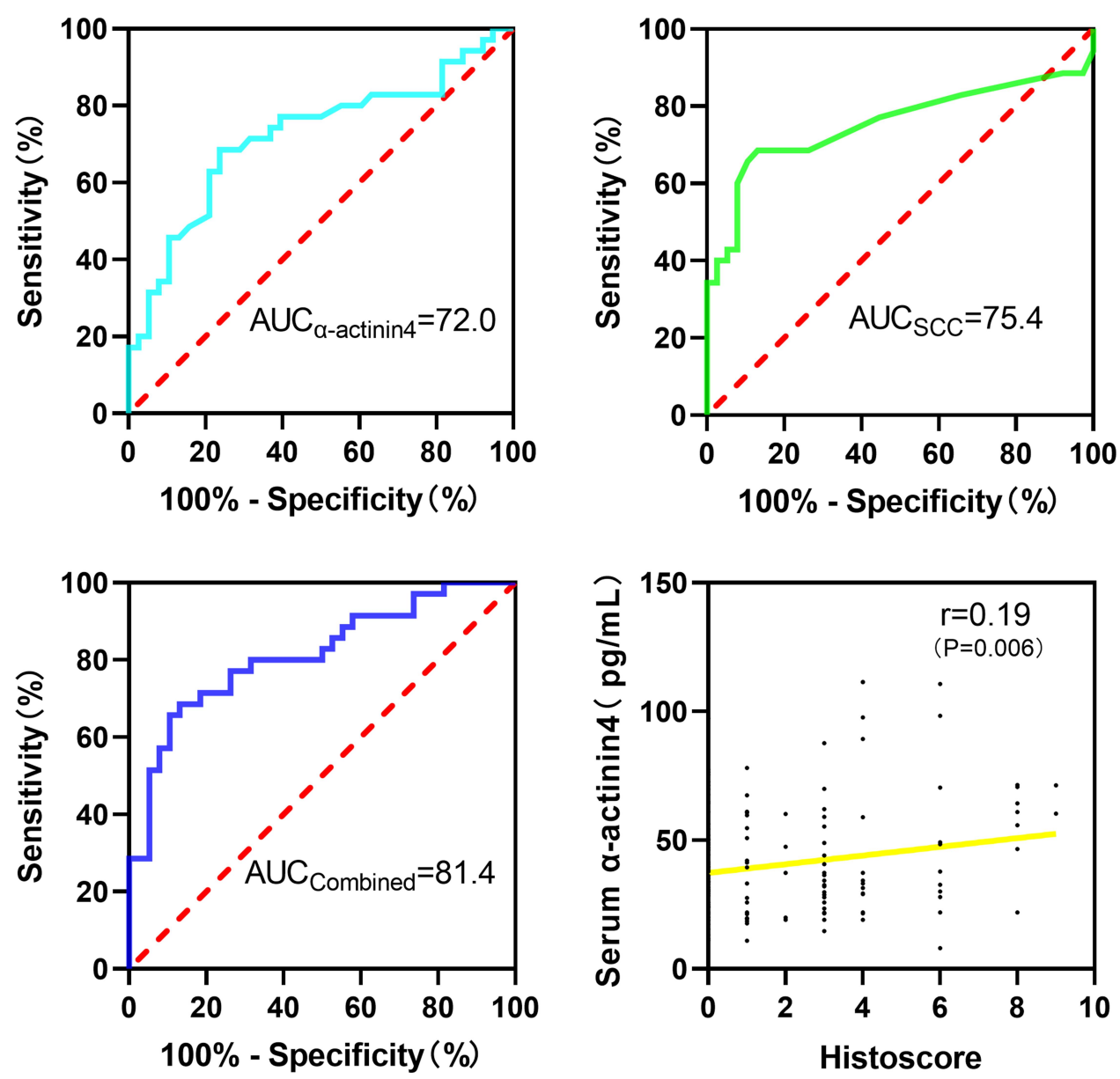

Figure 4 ROC curve analysis of different serum biomarkers for identifying CIN3+ disease and the correlation of ACTN4 expression in cervical tissue and serum with CIN3 + disease.

Abbreviations: ROC, receiver operating characteristic; AUC, area under the ROC curve.

SCC levels have been detected in $28-88 \%$ of cervical squamous cell carcinomas. ${ }^{21}$ The study of two or more biological markers in serum to increase the sensitivity and specificity of early screening of malignant tumours by making up their own blind spots and increasing their own superior detection ability has been a research hotspot of generations of scholars. In this study, ROC analysis suggested that ACTN4 could be a useful marker with $76.3 \%$ sensitivity and $94 \%$ specificity for discriminating CIN3 or worse patients from controls via a cut-off value of $33.07 \mathrm{pg} / \mathrm{mL}$. Interestingly, in combination with $\mathrm{SCC}-\mathrm{Ag}$, the sensitivity and specificity increased to $75.6 \%$ and $87.5 \%$, respectively. $\mathrm{CC}$ is a heterogeneous disease; thus, it is unlikely that any single marker will have a sufficiently high sensitivity.

In this study, we collected 39 cervical cancer tissues and 39 control tissues, and it is interesting to note that ACTN4 mRNA was not only overexpressed in CC tissue, but was also associated with a larger tumour size, advanced FIGO staging, and lymph node metastasis-positive cases. To further investigate the relationship between ACTN4 expression in cervical lesions and different cervical severity, we performed an IHC assay and found that ACTN4 expression strongly increased from NLIM to CC.H-T. An et $\mathrm{al}^{12}$ reported that ACTN4 has important roles in tumour growth and the change to the mesenchymal phenotype, consistent with our study. Unfortunately, our results show that ACTN4 mRNA expression is not related to HPV type16 infection, which is not consistent with a report from Qiqi Wang et al ${ }^{15}$ that showed that HPV16 E6 promoted cytoskeleton assembly and cell invasion via ACTN4. This may have been due to our use of cervical tissue collected from the female reproductive tract, which is full of various bacteria and is different from cervical cell models. Interestingly, a serological ACTN4 assay was performed on 4 patients with cervical ADC, and all of them exceeded the cut-off value $(33.12,34.31,35.41$, and $36.13 \mathrm{pg} / \mathrm{mL})$, while the expression in tissue was moderate (Figure 3 ). Although few 
patients with cervical ADC were included in our study, it is worthwhile to continue to collect a large number of specimens to evaluate the screening performance of ACTN4 for early cervical ADC. The qPCR results showed that ACTN4 mRNA was associated with the patient's FIGO stage, tumour size and lymph node metastasis, which can be further applied in the clinic to predict the progression of CC.

Several shortcomings in the present study should be acknowledged. First, the sample size was relatively small, and patients with FIGO III or FIGO IV were not selected in our study due to the relatively shorter period. We need to detect ACTN4 levels in patients with advanced CC in a future study. Second, the present study was a preliminary study that explored the clinical significance of serum ACTN4 for the early diagnosis of CC. Third, we need to perform experiments in vitro and vivo to further verify the relationship between ACTN4 and SCC-Ag. We found that overexpression of ACTN4 might contribute to the aggressive progression of $\mathrm{CC}$, but the molecular mechanisms underlying the oncogenic function of ACTN4 in CC remain poorly defined. Therefore, further in vitro and in vivo experiments should be designed to address these tissues.

\section{Conclusions}

Our study found that ACTN4 levels were overexpressed not only in blood from patients with CIN3 or worse, but also in their cervical tissues. ACTN4 mRNA is overexpressed in CC tissues and in tissues with advanced FIGO stage, larger tumour sizes and positive lymph node metastasis. ACTN4 in combination with SCC-Ag has a potential role as a biomarker for the diagnosis and prognosis of patients with CIN3 or worse.

\section{Abbreviations}

SCC-Ag, squamous cell carcinoma antigen; $\mathrm{CC}$, cervical cancer; HSIL, high-grade squamous intraepithelial lesion; NILM, negative for intraepithelial lesion or malignancy; FIGO, International Federation of Gynecology and Obstetrics; CIN, cervical intraepithelial neoplasia; ADC, cervical adenocarcinoma; PPV, positive predictive value; NPV, negative predictive value; PLR, positive likelihood ratio; NLR, negative likelihood ratio; YI, Youden index; ROC, receiver operating characteristic; AUC, area under the ROC curve.

\section{Ethics Statement}

This research has been carried out in accordance with the World Medical Association Declaration of Helsinki. This study received a documented review and approval from a formally constituted review board (the ethics committee of Fujian Medical University Union Hospital, 2017QH024). All patients and healthy volunteers provided written informed consent prior to their inclusion in the study.

\section{Acknowledgments}

The authors would like to thank Fujian Medical University Union Hospital for the fresh frozen, paraffin-embedded tumour and blood samples. Above all, we are grateful to the patients who made the study possible.

\section{Author Contributions}

All authors made a significant contribution to the work reported, whether it was in the conception, study design, execution, acquisition of data, analysis and interpretation, or in all these areas. All of the authors took part in drafting, revising or critically reviewing the article and gave final approval of version to be published. All the authors have agreed on the journal to which the article has submitted and agree to be accountable for all aspects of the work.

\section{Funding}

This work was supported by the Young and Middle-aged Teachers Fund, Education Department of Fujian province (JT180176); the Start-up Fund for Scientific Research, Fujian Medical University (2019QH1038); Joint Funds for the Innovation of Science and Technology, Fujian Province (2017Y9051); Fujian Medicine Innovation Program, (2017-CX-20 and 2018-CX-18); Fujian Undergraduate Education and Teaching Reform Project (FBJG20180225) and Key Project of Educational Reform in Fujian Medical University (J18002).

\section{Disclosure}

The authors report no conflicts of interest regarding this work.

\section{References}

1. Ferlay J, Soerjomataram I, Dikshit R, et al. Cancer incidence and mortality worldwide: sources, methods and major patterns in GLOBOCAN 2012. Int $J$ Cancer. 2015;136(5):E359-386. doi:10.1002/ijc. 29210

2. de Sanjosé S, Diaz M, Castellsagué X, et al. Worldwide prevalence and genotype distribution of cervical human papillomavirus DNA in women with normal cytology: a meta-analysis. Lancet Infect Dis. 2007;7(7):453-459. doi:10.1016/S1473-3099(07)70158-5

3. Dickson EL, Vogel RI, Geller MA, Downs LS. Cervical cytology and multiple type HPV infection: a study of 8182 women ages 31-65. Gynecol Oncol. 2014;133(3):405-408. doi:10.1016/j.ygyno.2014.03. 552 
4. Cohen PA, Jhingran A, Oaknin A, Denny L. Cervical cancer. Lancet. 2019;393(10167):169-182. doi:10.1016/S0140-6736(18)32470-X

5. Schiffman M, Doorbar J, Wentzensen N, et al. Carcinogenic human papillomavirus infection. Nat Rev Dis Primers. 2016;2:16086.

6. Arbyn M, Ronco G, Anttila A, et al. Evidence regarding human papillomavirus testing in secondary prevention of cervical cancer. Vaccine. 2012;30(Suppl 5):F88-99. doi:10.1016/j.vaccine.2012.06.095

7. Valenti G, Vitale SG, Tropea A, Biondi A, Lagana AS. Tumor markers of uterine cervical cancer: a new scenario to guide surgical practice? Updates Surg. 2017;69(4):441-449. doi:10.1007/s13304-017-0491-3

8. Honda K. The biological role of actinin-4 (ACTN4) in malignant phenotypes of cancer. Cell Biosci. 2015;5(1):41. doi:10.1186/s13578015-0031-0

9. Honda K, Yamada T, Hayashida Y, et al. Actinin-4 increases cell motility and promotes lymph node metastasis of colorectal cancer. Gastroenterology. 2005;128(1):51-62. doi:10.1053/j.gastro.2004.10.004

10. Kikuchi S, Honda K, Tsuda H, et al. Expression and gene amplification of actinin-4 in invasive ductal carcinoma of the pancreas. Clin Cancer Res. 2008;14(17):5348-5356. doi:10.1158/1078-0432.CCR-08-0075

11. Yamamoto S, Tsuda $\mathrm{H}$, Honda $\mathrm{K}$, et al. Actinin-4 expression in ovarian cancer: a novel prognostic indicator independent of clinical stage and histological type. Mod Pathol. 2007;20(12):1278-1285. doi:10.1038/modpathol.3800966

12. An HT, Yoo S, Ko J. Alpha-actinin-4 induces the epithelial-tomesenchymal transition and tumorigenesis via regulation of snail expression and beta-catenin stabilization in cervical cancer. Oncogene. 2016;35(45):5893-5904. doi:10.1038/onc.2016.117

13. Wang Q, Qin Q, Song R, et al. NHERF1 inhibits beta-cateninmediated proliferation of cervical cancer cells through suppression of alpha-actinin-4 expression. Cell Death Dis. 2018;9(6):668. doi:10.1038/s41419-018-0711-x
14. Jung J, Kim S, An HT, Ko J. $\alpha$-actinin-4 regulates cancer stem cell properties and chemoresistance in cervical cancer. Carcinogenesis. 2020;41(7):940-949. doi:10.1093/carcin/bgz168

15. Wang Q, Song R, Zhao C, et al. HPV16 E6 promotes cervical cancer cell migration and invasion by downregulation of NHERF1. Int J Cancer. 2019;144(7):1619-1632.

16. Starodubtseva NL, Brzhozovskiy AG, Bugrova AE, et al. Label-free cervicovaginal fluid proteome profiling reflects the cervix neoplastic transformation. J Mass Spectrom. 2019;54(8):693-703. doi:10.1002/ jms. 4374

17. Van Raemdonck GA, Tjalma WA, Coen EP, Depuydt CE, Van Ostade XW. Identification of protein biomarkers for cervical cancer using human cervicovaginal fluid. PLoS One. 2014;9(9):e106488. doi:10.1371/journal.pone.0106488

18. Van Ostade X, Dom M, Tjalma W, Van Raemdonck G. Candidate biomarkers in the cervical vaginal fluid for the (self-)diagnosis of cervical precancer. Arch Gynecol Obstet. 2018;297(2):295-311. doi:10.1007/s00404-017-4587-2

19. Fang C, Li JJ, Deng T, Li BH, Geng PL, Zeng XT. Actinin-4 as a diagnostic biomarker in serum of breast cancer patients. Med Sci Monit. 2019;25:3298-3302. doi:10.12659/MSM.912404

20. Liu Z, Shi H. Prognostic role of squamous cell carcinoma antigen in cervical cancer: a meta-analysis. Dis Markers. 2019;2019:6710352. doi: $10.1155 / 2019 / 6710352$

21. Guo Q, Zhu J, Wu Y, et al. Predictive value of preoperative serum squamous cell carcinoma antigen (SCC-Ag) level on tumor recurrence in cervical squamous cell carcinoma patients treated with radical surgery: a single-institution study. Eur J Surg Oncol. 2019;313(7068):1285-1290.
Risk Management and Healthcare Policy

\section{Publish your work in this journal}

Risk Management and Healthcare Policy is an international, peerreviewed, open access journal focusing on all aspects of public health, policy, and preventative measures to promote good health and improve morbidity and mortality in the population. The journal welcomes submitted papers covering original research, basic science, clinical \& epidemiological studies, reviews and evaluations, guidelines, expert opinion and commentary, case reports and extended reports. The manuscript management system is completely online and includes a very quick and fair peer-review system, which is all easy to use. Visit http://www.dovepress.com/testimonials.php to read real quotes from published authors. 